\section{* address correspondence to kai.dallmeier@kuleuven.be} Leuven, Leuven, Belgium.

Biology, KU Leuven, Leuven, Belgium. Ghana. 8 Belgium. Tropical Medicine, Antwerp, Belgium. \section{列}

\# equal contribution

\title{
Biodistribution and Environmental Safety of a Live-attenuated YF17D-vectored SARS-CoV-2 Vaccine Candidate
}

\section{Li-Hsin Li 1, Laurens Liesenborghs 1,6\#, Lanjiao Wang 2\#, Marleen Lox ${ }^{3}$, Michael}

Bright Yakass ${ }^{1,4}$, Sander Jansen ${ }^{1}$, Ana Lucia Rosales Rosas ${ }^{2}$, Xin Zhang ${ }^{1}$, Hendrik Jan Thibaut ${ }^{5}$, Dirk Teuwen ${ }^{1}$, Johan Neyts ${ }^{1}$, Leen Delang ${ }^{2}$, and Kai Dallmeier ${ }^{1 *}$

${ }^{1}$ Department of Microbiology, Immunology and Transplantation, Rega Institute, Virology and Chemotherapy, Molecular Vaccinology and Vaccine Discovery, KU

${ }^{2}$ Department of Microbiology, Immunology and Transplantation, Rega Institute, Virology and Chemotherapy, Mosquito Virology Team, KU Leuven, Leuven, Belgium.

${ }^{3}$ Department of Cardiovascular Diseases, Centre for Molecular and Vascular

${ }^{4}$ West African Centre for Cell Biology of Infectious Pathogens (WACCBIP),

4 Department of Biochemistry, Cell and Molecular Biology, University of Ghana, Accra,

${ }^{5}$ Department of Microbiology, Immunology and Transplantation, Rega Institute,

Translational Platform Virology and Chemotherapy (TPVC), KU Leuven, Leuven,

${ }^{6}$ The Outbreak Research Team, Department of Clinical Sciences, Institute of 


\section{ABSTRACT}

27 New platforms are urgently needed for the design of novel prophylactic vaccines and 28 advanced immune therapies. Live-attenuated yellow fever vaccine YF17D serves as vector for several licensed vaccines and platform for novel vaccine candidates. Based on YF17D, we developed YF-S0 as exceptionally potent COVID-19 vaccine candidate. However, use of such live RNA virus vaccines raises safety concerns, i.e., adverse events linked to original YF17D (yellow fever vaccine-associated neurotropic; YELAND, and viscerotropic disease; YEL-AVD). In this study, we investigated the biodistribution and shedding of YF-S0 in hamsters. Likewise, we introduced hamsters deficient in STAT2 signaling as new preclinical model of YEL-AND/AVD. Compared to parental YF17D, YF-S0 showed an improved safety with limited dissemination to brain and visceral tissues, absent or low viremia, and no shedding of infectious virus. Considering yellow fever virus is transmitted by Aedes mosquitoes, any inadvertent exposure to the live recombinant vector via mosquito bites is to be excluded. The transmission risk of YF-S0 was hence evaluated in comparison to readily transmitting YFV-Asibi strain and non-transmitting YF17D vaccine, with no evidence for productive infection of vector mosquitoes. The overall favorable safety profile of YF-SO is expected to translate to other novel vaccines that are based on the same YF17D platform. 


\section{INTRODUCTION}

Roughly two years after first emergence in 2019/2020, more than 5 million people have succumbed to Coronavirus Disease 2019 (COVID-19) caused by Severe Acute Respiratory

Syndrome

Coronavirus 2 (SARS-CoV-2) (https://coronavirus.jhu.edu/map.html). Mass immunization is key to mitigating the expanding pandemic [1]. A set of rapidly developed prophylactic vaccines plays a crucial role in global immunization against SARS-CoV-2. Several of these vaccines are first-in-class based on novel platforms, including game changer mRNA vaccines and viral vector vaccines that are unprecedented in both, their high clinical efficacy as well as the incremental advance in breakthrough innovation [2-4]. However, a global vaccine supply shortage, the dependence on an ultra-cold chain system in case of mRNA vaccines, and the continuous emergence of virus variants pose unmet challenges [5, 6]. Unfortunately, long-term effectiveness of current SARS-CoV-2 vaccines is waning due to the combined effect of $(I)$ a rapid decay of virus-neutralizing antibodies ( $\mathrm{nAb}$ ) over time and (ii) emergence of new variants escaping vaccineinduced immunity [7-9]. Furthermore, several first-generation COVID-19 vaccines have a rather high reactogenicity. With the growing number of vaccinated people, more cases and a wider spectrum of adverse effects following immunization (AEFI), including severe adverse effects (SAE) such a myocarditis or life-threatening deepvenous thrombosis are described [10-15]. In summary, there is an urgent to develop new and improved second-generation COVID-19 vaccines to quench the pandemic.

Recently, we used an alternative vaccine platform that uses the fully replication competent live-attenuated yellow fever vaccine YF17D as vector [16] and developed a virus-vectored SARS-CoV-2 vaccine candidate (YF-SO) that expresses a stabilized prefusion form of SARS-CoV-2 spike protein (S0) [17]. YF-S0 was shown to induce vigorous humoral and cellular immune responses in hamsters (Mesocricetus auratus), mice (Mus musculus) and cynomolgus macaques (Macaca fascicularis) and was able to prevent COVID19-like disease after single-dose vaccination in a stringent hamster model. Due to its YF17D backbone, YF-S0 could serve as dual vaccine to also prevent yellow fever virus (YFV) infections, which should provide an added benefit for populations living in regions at risk of YFV outbreaks [18]. 
In addition to preclinical efficacy, development of such a new vaccine requires in-depth evaluations of its safety to support progression from preclinical study to clinical trials. In particular for live-attenuated viral vaccines such as YF-S0, the biodistribution of the vaccine virus after administration needs to be assessed [19] to understand the viral organ tropism and hence to exclude potential direct harm to specific tissues. Our vaccine candidate YF-SO showed an excellent safety profile in multiple preclinical models, including in NHP as well as in interferon-deficient mice and hamsters [17]. However, use of such a recombinant YF17D vaccine entails some potential concerns [19]. Particularly, replication and persistence of YF-S0 in tissues and body fluids poses a theoretical risk of YF vaccine-associated viscerotropic disease (YEL-AVD) and YF vaccine-associated neurotropic disease (YEL-AND), which are originally linked to parental YF17D [20]. Regarding to this, the parental YF17D vaccine are commonly used as benchmark for direct comparison in safety assessment [19].

Here, we investigated the biodistribution and shedding of YF-SO following vaccination in hamsters, with as aim to understand ( $I$ ) to what extent YF-SO causes viremia resulting into virus dissemination to vital organs; (ii) to evaluate the risks of YF-SO for YEL-AVD/AND by confirming its transient and self-limited replication in vivo [17], restricting the risks for YEL-AVD/AND ; (iii) to what extent viral RNA remains detectable in body secretions and, in case, (iv) if this poses any environment risks for shedding of recombinant infectious virus. Furthermore, YFV is also a mosquito-borne virus. To eliminate the concerns that YF-S0, which employs licensed YF17D as a vector and hence, despite being proven highly attenuated, might lead to an increased environmental risk causing by phenotypical change as any other recombinant viruses. Taking this theoretical consideration into account, we tested the infectivity of YF-SO on Aedes aegypti (Ae. aegypti) mosquitoes to assess its transmission potential. Ae. aegypti was selected as target mosquito species because of its well-known high vector competence for YFV [21]. It is well documented that wild-type YF-Asibi can infect and disseminate in Ae. aegypti while YF17D only occasionally infects the midgut and is unable to disseminate to secondary organs [22, 23]. Therefore, these two YFV strains were used as controls to assess transmission of YF-SO by a competent vector. 
107 Finally, we corroborate the favorable safety profile of YF-S0 by reporting limited

108 dissemination and shedding in vaccinated hamsters, nor any risk of mosquito-borne 109 transmission.

110 


\section{RESULTS}

112 Tissue distribution of YF-S0 and parental YF17D in hamsters

113 For our assessment, we chose wild-type (WT) Syrian golden hamsters as preferred 114 small animal model of YFV infection [24] and injected them with a high dose (104 PFU) 115 of either YF17D $(n=6)$ or YF-S0 $(n=6)$ via intraperitoneal route to achieve maximal 116 exposure; with primary pharmacodynamics documented before [17] and confirmed 117 here by consistently high seroconversion rates (at least 80\%) to YFV-specific nAb (Fig. 118 S1). As methods control, we inoculated STAT2-knockout (STAT2 ${ }^{-/-}$) hamsters with $10^{4}$ 119 PFU of YF17D $(\mathrm{n}=2)$. STAT2 ${ }^{-/-}$hamsters are deficient in antiviral type I and type III 120 interferon responses [25] and therefore prone to uncontrolled flavivirus replication [26].

121 Tissues sampled for analysis were chosen based on biodistribution data available from non-human primates and humans. In macaques, detection of YF17D RNA has been reported in lymph nodes, spleen and liver at 7 days post subcutaneous inoculation

124 [27]. Likewise, viral RNA is widespread and abundantly found in spleen, liver, brain, 125 kidney, and other organs in patients who developed YEL-AVD [20, 28]. Based on this 126 knowledge, we collected spleen, liver, brain, and kidney as most common target organs to assess the risks for YEL-AVD and YEL-AND. Ileum and parotid gland were collected as additional excretory tissues, and lung as main target of COVID19 (Fig. 1A). From our previous experience [17], we observed that the replication of YF17D or YF-SO is transient and well tolerated in WT hamsters. Tissue analysis in hamsters was thus performed 7 days post inoculation (dpi), i.e., few days after peak of viremia and at a timepoint which STAT2 ${ }^{-/-}$hamsters needed to be euthanized for humane reasons.

Viral RNA above detection limits in YF17D vaccinated WT hamsters was mostly limited to spleen (4/6), with exception of a single hamster in which viral RNA was widespread to brain, parotid gland, and lung (Fig. 1B and Suppl Table 1). Detection of YF-S0 was markedly less frequent and restricted to only kidney (2/6) and lung (1/6) (Fig. 1B and

137 Fig. 1D). Overall, in either group RNA level was low and barely detectable by sensitive 138 RT-qPCR, indicative for limited replication in WT hamsters. In contrast, unrestricted 139 replication of virus to high viral loads was observed in STAT2 ${ }^{-/-}$hamsters (Fig. 1B and 140 Fig. 1C). Importantly, no viral RNA nor infectious virus could be detected in brains of 141 YF-S0 vaccinated hamsters, suggesting a low associated YEL-AND risk (Fig. 1D and 142 Fig. 1E). 
143 Viremia is considered a key indicator for the risk of developing YEL-AVD. Kinetics of

144 viral RNA in serum as proxy for viremia have been reported earlier for WT hamsters 145 vaccinated with YF17D or YF-S0 [17] and are discussed here in comparison to 146 respective data from STAT2-/- controls (Fig. 2B). Viremia can be detected consistently 147 in all YF17D vaccinated WT hamsters (6/6) starting at $1 \mathrm{dpi}$ and lasting for 2.5 (1-4) 148 days in median (95\% confidence interval); by contrast, viral RNA was detected only 149 once at 3 dpi in a single YF-S0 vaccinated hamster (1/6) (Fig. 2B and Suppl. Table 2). 150 In STAT2 ${ }^{-/-}$hamsters, YF17D grew unrestrictedly to markedly increased viral RNA 151 levels (Fig. 2B), readily detectable by virus isolation (Fig. S2). Integration of data over 152 the course of immunization (area under the curve, AUC) indicated a significant reduced overall serum virus load in YF-S0 vaccinated animals (Fig. 2F).

\section{Limited shedding of YF-S0 and parental YF17D RNA}

Since shedding of viral RNA in urine after YF17D vaccination has been reported [29], we sampled different body fluids to investigate respective virus levels (Fig. 2A). Within all longitudinally sampled specimens, viral RNA was detected only sporadically in urine $(1 / 56 ; 3 / 58)$, faeces $(2 / 65 ; 1 / 66)$, and buccal swabs $(1 / 66 ; 3 / 66)$ of both YF17D and YF-S0 vaccinated hamsters, mostly at very low copy numbers, and not linked to viremia (Figure 2C-2E, Fig. S3, and Suppl Table 3-5). Noteworthy, viral RNA could only be detected, if at all, only within the first $11 \mathrm{dpi}$, clearly indicating that viral replication was self-limiting, leading to the final elimination of the live viral vector from all tissues. Also, there was no significant difference regarding the AUC between both groups (Fig. 2G-2I). The potential risk of YF-SO to be spread by excrements of vaccinated individuals should hence be as low as for YF17D. In addition, no viable virus could be isolated from urine samples with RNA counts as high as $10^{8}$ copies $/ \mathrm{mL}$ (not shown), in line with no clinical evidence for secondary spread in urine, matching longstanding field experience for YF17D.

Abortive infection of YF-S0 on yellow fever virus competent vector Ae. aegypti competent mosquito vector could theoretically lead to unintentional exposure to the 
174 YF-S0 should be excluded regarding main indicators of mosquito vector competence 175 [21, 31, 32] (Fig. 3A): (i) sufficient virus ingestion from infectious blood meal; (ii) 176 productive infection of virus in mosquito midgut (midgut infection barrier, MIB); and, 177 (iii) virus escapes from midgut barrier (MEB), i.e., dissemination to parenteral tissues 178 to establish sufficiently high virus loads in salivary glands to enable transmission. To 179 this end, Aedes aegypti mosquitoes, as the species of YFV competent vector [21], 180 were given infectious blood meals with either no virus, YF17D, YF-S0 or wild-type YF181 Asibi strain as positive control $[22,23]$. Infection was determined by RT-qPCR and virus isolation on day 0 on whole mosquitoes (ingestion step), on day 14 in thorax and abdomen (virus infection and replication in mosquito midgut; marked as main body), and on day 14 dissemination in head, legs and wings (dissemination).

185 Experimental feeding was equally efficient for all three virus groups regarding both viral 186 RNA and infectious virus recovered (Fig. 3B\&C). However, 14 days after feeding, viral 187 RNA was detected exclusively in specimens from the YF17D group (8/15) and YF188 Asibi group (8/23); yet none from the YF-S0 group. Importantly, infectious viral particles 189 were only detectable in the YF-Asibi group, with virus loads as high as about $10^{6}$ $190 \mathrm{TCID}$ 50/body on average (Fig. 3C). For dissemination beyond the MEB, the remaining 191 head, legs and wings of each six virus-positive mosquitoes with highest body virus 192 loads from the YF17D and YF-Asibi groups, respectively, and six randomly chosen 193 specimens from the YF-S0 group were evaluated. All these specimens from the YF194 Asibi group (6/6) scored positive for dissemination, while none from the YF-S0 or 195 YF17D groups (Fig. 3B-C). The results showing that YF-S0 is neither able to pass the 196 MIB for midgut infection, nor to escape from the midgut (MEB) for dissemination (Fig. 3D\&E). 


\section{DISCUSSION}

200 The live-attenuated YF17D vaccine is considered as one of the most powerful and 201 successful vaccines and has been used on humans for decades [33]. Its well-known characteristics of stimulating both vigorous humoral and cellular immune responses, as well as favorable innate responses is of interest for other vaccine targets using the YF17D genome as a backbone [16]. We recently generated a particularly potent YF17D-vectored vaccine candidate, YF-S0, against SARS-CoV-2 infection, inserting the non-cleavable spike protein of SARS-CoV-2 (S0) between the $E$ and NS1 region of YF17D [17]. This construct serves as antigens to induce vigorous immune responses against both

SARS-CoV-2

and

YFV

infections

[17].

Apart from YF-S0, YF17D is currently the only fully replication competent viral vector that is part of any licensed recombinant live viral vaccine in wide use for human medicine; i.e. in the two licensed human vaccines, JE-CV (against Japanese encepahilitis; Imojev ${ }^{\circledR}[34]$ ) and CYD-TDV (against all four serotypes of dengue virus; Dengvaxia® [35]). Additional YF17D-based vaccine candidates are in different stages of (pre)clinical developed, including vaccines against other flaviviruses (West Nile virus: ChimeriVax-WN02 [36]; Zika virus: YF-ZIKprM/E [37]) or non-flaviviruses (HIV: rYF17D/SIVGag45-269 [38]; Lassa virus: YFV17D/LASVGPC [39]; chronic hepatitis $B$ virus: YF17D/HBc-C [40]). As these YF17D-vectored vaccines has been proved, YFS0 also trigger vigorous protective immune responses, including high levels of SARSCoV-2 neutralizing antibodies after a single dose vaccination in hamsters, mice and cynomolgus macaques. However, despite little (pre)clinical evidence nor such reports from post-marketing surveillance, all these YF17D-vectored vaccines share the theoretical concerns of the SAEs associated with the parental YF17D vaccines, such as YEL-AVD (0.4 per 100,000) and YEL-AND (0.8 per 100,000) [19, 20, 41, 42].

To temper the remaining safety concerns, the viscerotropism and neurovirulence of YF-S0 was compared head-to-head with parental YF17D virus by investigating the biodistribution and viremia following administration of either vaccine virus in hamsters. We demonstrate that parental YF17D can spread systemically and viral RNA can be detected in spleen, brain, parotid gland, and lung in YF17D vaccinated WT hamsters. However, replication of YF17D remains restricted, resulting in infectious virus loads 
232 below detection limits. Compared to YF17D, detection of YF-S0 was further limited, with minute amounts of viral RNA in kidney and lung. Unrestricted virus replication to

234 high viral loads as cause of viscerotropic or neurotropic disease was observed only in $\mathrm{STAT2}^{-/-}$hamsters, in line with the essential role innate interferon signaling plays in live vaccines [30,43] and control of viral infections in general [44]. In addition, in YFS0 vaccinated WT hamsters, detection of viremia was rare (Fig. 2B) and importantly, less frequent (1/6) and markedly lower in magnitude (AUC) and duration (1 day) compared to parental YF17D (6/6 for $>2$ days). Taken together, the overall limited tissue distribution of YF-SO as well as the low abundance of its RNA in blood, below detection limits for infectious virus, suggest a further lowered risk of YEL-AVD/AND for YF-S0 than that reported parental YF17D. To further investigate the potential environment risk associated with shedding of recombinant virus, we collected urine, faeces and buccal swabs from vaccinated hamsters and checked for the presence of viral RNA for 29 days to determine how long YF-S0 would remain detectable in body secretions as compared to YF17D. No significant differences in vaccine RNA shedding were observed between YF17D and YF-S0 during the course of immunization (Fig. 2G-2I, AUC). Importantly, no infectious virus could be isolated, suggesting the risk is very low, even if any inadvertent exposure by vaccinated individuals to their environment. In summary, these results obtained in a hamster model of YF17D vaccination clearly demonstrate that $(I)$ the overall viral tissue burden for YF-S0 was considerably lower than for parental YF17D, and (ii) presence of viral RNA in body secretions (urine, feces, and buccal swab) was equally low as for YF17D, mostly likely void of residual infectious virus particles. YF-SO vaccine virus infection is transient and harbors minimal, if at all any, risk of shedding nor evidence for environmental biosafety concern.

Last, though the chances of YF17D-vectored vaccines to be transmitted by arthropod vectors are minimal, we evaluated the replication competence of YF-S0 in yellow fever mosquito vector (i.e., Ae. aegypti). While parental YF17D passed the MIB and got restricted at the MEB as previous documented [22, 23], YF-S0 was already blocked at the first barrier with no remaining viral RNA or infectious virus detectable after an infectious bloodmeal. Hence, the transmissibility of YF-SO by mosquitoes is to be considered neglectable. 
265 Altogether, YF-SO is considered a safe and efficacious vaccine candidate for the 266 prevention of COVID19. A similar improved safety as compared to parental YF17D can 267 be expected for other vaccines following the same design principle, i.e., using 268 transgenic, yet fully replication-competent YF17D as vector [16, 40]. 


\section{Funding}

273

274 This project has received funding from the European Union's Horizon 2020 Research

275 and Innovation Program under grant agreement no. 733176 (RABYD-VAX to K.D. and

276 J.N.), and no. 101003627 (SCORE to J.N.). Funding was further provided by the

277 Research Foundation Flanders (FWO) under the Excellence of Science (EOS)

278 program (no. 30981113, VirEOS to K.D and J.N.), the FWO COVID19 call (no.

279 G0G4820N), and by the KU Leuven/UZ Leuven COVID-19 Fund (COVAX-PREC

280 project). L.H.L. acknowledges support by a PhD scholarship grant from the KU Leuven

281 Special Research Fund (DBOF/14/062). L.L. is member of the Institute of Tropical

282 Medicine's Outbreak Research Team which is financially supported by the Department 283 of Economy, Science and Innovation (EWI) of the Flemish Government. X.Z. was 284 supported by a PhD scholarship grant from the China Scholarship Council (CSC, no. 285 201906170033). L.D. received funding from KU Leuven Internal Funds (C22/18/007 286 and STG/19/008), as well as K.D. (C3/19/057 Lab of Excellence). This publication was 287 supported by the Infravec2 project, which has received funding from the EU's Horizon 2882020 Research and Innovation Program 2020 (grant agreement no. 731060). 


\section{Contributions}

291 Overall conceptual design: L.-H.L., L.L., D.T., and K.D.; methodology: L.-H.L., L.L., 292 L.W.; in vivo experiments (hamsters): L.L. and M.L.; in vivo experiments (mosquitoes): 293 L.W. and A.L.R.R.; in vitro experiments: L.-H.L. and X.Z.; in vitro experiment 294 (serology): H.J.T.; design and generating of YF-Asibi: M.B.Y. and S.J.; data 295 management and analysis: L.-H.L. and L.L.; writing of manuscript- draft: L.-H.L.; writing 296 of manuscript- review and editing: L.-H.L., L.L., L.W., L.D., D.T., and K.D.; supervision: 297 J.N. and K.D.; funding acquisition: J.N. and K.D.

\section{Acknowledgements}

299 We thank Thibault Francken, Dagmar Buyst, Niels Cremers, Birgit Voeten, and Jasper 300 Rymenants for their technical support on specimens' preparation and virus titration as 301 well as Jasmine Paulissen for her technical assistance on generating serology data.

\section{Data availability}

303 All data supporting the findings in this study are available from the corresponding 304 author upon request.

\section{Competing interests}

306 The authors declare that there are no competing interests. This manuscript is 307 currently under peer review. 


\section{Materials and Methods}

\section{Animal experiment}

\section{Hamsters}

313 Wild-type (WT) outbred specific pathogen-free Syrian hamsters (Mesocricetus 314 auratus) were purchased from Janvier Laboratories, France. The generation [45] and characterization [25] of STAT2 ${ }^{-/-}$(gene identifier: 101830537) hamsters has been described elsewhere. STAT2 ${ }^{-/-}$hamsters were bred in-house. Hamsters (max. $n=2$ ) were housed in individually ventilated cages (Sealsafe Plus, Tecniplast; cage type GR900), under standard conditions of $21^{\circ} \mathrm{C}, 55 \%$ humidity and $12: 12$ light:dark cycles. Hamsters were provided with food and water ad libitum, as well as extra bedding material and wooden gnawing blocks for enrichment as previously described. This project was approved by the KU Leuven ethical committee (P015-2020), following institutional guidelines approved by the Federation of European Laboratory Animal Science Associations (FELASA). Hamsters were euthanized by intraperitoneal administration of $500 \mu \mathrm{L}$ (hamsters) Dolethal $(200 \mathrm{mg} / \mathrm{mL}$ sodium pentobarbital, Vétoquinol SA).

\section{Vaccine and virus stocks}

327 Vaccine viruses used throughout this study have been described [17]. YF-S0 was 328 derived from a cDNA clone of YF17D (GenBank: X03700) with an in-frame insertion of 329 a non-cleavable version of the SARS-CoV-2 S protein (GenBank: MN908947.3) in the 330 YFV E/NS1 intergenic region. YF-S0 vaccine stocks were grown on baby hamster 331 kidney (BHK21) cells. The molecular and antigenic structure and replication of YF-S0 332 has been described in detail [17]. Original YF17D vaccine (Stamaril, Sanofi-Pasteur; 333 lot number G5400) was purchased via the pharmacy of the University Hospital Leuven 334 and passaged twice in Vero E6 cells prior to use. The construction and rescue of YF335 Asibi from an infectious cDNA clone will be described elsewhere (Yakass, Jansen et 336 al.). The respective YFV cDNA sequence was adjusted to match previously described molecular clone Ap7M [46]. All virus stocks were titrated by plaque assay on BHK21 cells [17]. 
340 WT hamsters (6-8 weeks old, female) were inoculated intraperitoneally with $10^{4}$ 341 PFU/mL dose of YF17D $(n=6)$ or YF-S0 $(n=6)$. STAT2 ${ }^{-/-}$hamster (6-8 weeks old, 342 female) were inoculated intraperitoneally with $10^{4} \mathrm{PFU} / \mathrm{mL}$ of YF17D $(\mathrm{n}=2)$. At $7 \mathrm{dpi}$, 343 blood, spleen, liver, brain, kidney, ileum, parotid gland, and lung were collected.

\section{Shedding}

345 WT hamsters (6-8 weeks old, female) were inoculated intraperitoneally with $10^{4}$ $346 \mathrm{PFU} / \mathrm{mL}$ of YF17D $(\mathrm{n}=6)$ or YF-S0 $(\mathrm{n}=6)$. STAT2 ${ }^{-/-}$hamsters $(6-8$ weeks old, male) 347 were inoculated intraperitoneally with $10^{4} \mathrm{PFU} / \mathrm{mL}$ of YF17D $(\mathrm{n}=3)$. Blood, urine, 348 faces, and buccal swab were collected daily for the first $5 \mathrm{dpi}$, then every other day 349 until $11 \mathrm{dpi}$ and 15, 22 (except for the blood) and $29 \mathrm{dpi}$, and afterwards once a week $350 \quad$ until 29 dpi.

\section{Mosquito experiment}

\section{Mosquito strain}

353 Ae. aegypti Paea [47] were obtained via the Infravec2 consortium 354 (https://infravec2.eu/product/live-eggs-or-adult-females-of-aedes-aegypti-strain-paea355 2/) from Institute Pasteur of Paris. Mosquitoes were maintained at the insectary of 356 Rega Institute, and the fourth generation was used for this study. In brief, larvae were fed with yeast tablets (Gayelord Hauser, France) until the pupae stage prior to transfer to cages for emergence. Adults were maintained with cotton soaked in $10 \%$ sucrose solution under standard conditions $\left(28^{\circ} \mathrm{C}, 80 \%\right.$ relative humidity, and $14 \mathrm{~h}: 10 \mathrm{~h}$ light/dark cycle).

\section{Oral infection and sample collection}

362 7-day-old female mosquitoes were starved $24 \mathrm{~h}$ prior to infection. Infectious blood meals contained rabbit erythrocytes plus $5 \mathrm{mM}$ adenosine triphosphate as

364 phagostimulant, supplemented with virus stocks to final titers of $2 \times 10^{5} \mathrm{PFU} / \mathrm{mL}$ for both

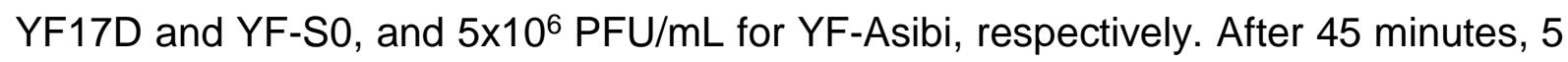
full engorged females from each group were frozen for viral input assessment (ingestion check, Fig. 3A), and the rest kept with $10 \%$ of sugar solution under both controlled conditions $\left(28 \pm 1^{\circ} \mathrm{C}\right.$, relative humidity of $80 \%$, light/dark cycle of $14 \mathrm{~h} / 10 \mathrm{~h}$, supplied with $10 \%$ sucrose solution) and BSL-3 containment conditions. At 14 dpi, 
370 mosquitoes were dissected into two parts; main body (thorax and abdomen) and remainder, collected individually in tubes containing PBS and $2.8 \mathrm{~mm}$ ceramic beads

372 (Precellys). The samples were homogenized and pass through $0.8 \mu \mathrm{m}$ column filters

373 (Sartorius, Germany). Thus, cleared supernatants were used for TCID50 assay or keep 374 at $-80^{\circ} \mathrm{C}$ for RNA extraction and subsequent RT-qPCR analysis.

\section{RNA extractions}

376 Solid tissues (organs), faeces and buccal swabs were homogenized in a bead mill

377 (Precellys) in lysis buffer (Macherey-Nagel; cat no. 740984.10). After homogenization, 378 samples were centrifuged at 10,000 rpm for 5 min to remove cell debris, and total RNA 379 was extracted by using NucleoSpin Plus RNA virus Kit (Macherey-Nagel, cat no. $380740984.10)$. For serum $(50 \mu \mathrm{l})$, urine $(50 \mu \mathrm{l})$ and homogenates of mosquito samples 381 (150 $\mu \mathrm{l})$, NucleoSpin RNA virus kit (Macherey-Nagel; cat no. 740956.250) was used 382 for RNA extraction.

\section{RT-qPCR}

384 RT-qPCR for YFV detection was performed as previously described [17] using primers and probe targeting the YFV NS3 gene [23] on an ABI 7500 Fast Real-Time PCR System (Applied Biosystems). Absolute quantification was based on standard curves generated from 5-fold serial dilutions of YF17D cDNA with a known concentration.

\section{TCID 50 assay}

389 For virus isolation and quantification BHK21 cells were infected with 10-fold serial 390 dilutions in 96-well plates, and incubated at $37^{\circ} \mathrm{C}$ for 6 days using DMEM with $2 \%$ fetal 391 bovine serum (Hyclone), 2 mM L-glutamine (Gibco), 1\% sodium bicarbonate (Gibco), and $1 \%$ antibiotics (PenStrep) as assay medium. Solid tissues were homogenized in 393 a bead mill (Precellys) in assay medium, and centrifuged at $10,000 \mathrm{rpm}$ for $5 \mathrm{~min}\left(4^{\circ} \mathrm{C}\right)$ 394 to remove debris. Resulting viral titers were calculated by the Reed and Muench 395 method.

\section{Serum neutralization test (SNT)}

397 Titers of YFV-specific neutralizing antibodies were determined using BHK21 cells and 398 a mCherry-tagged variant of YF17D virus (YFV-mCherry) as described [17]. In brief, 
399 YFV-mCherry was mixed and incubated with serial diluted of sera for $1 \mathrm{~h}$ at $37^{\circ} \mathrm{C}$, and 400 subsequently transferred to BHK21 cells grown in 96-well plates for infection. At 3 days 401 post infection, the relative infection rate was quantified by counting mCherry402 expressing cells versus total cells on a high content screening platform (CX5, Thermo 403 Fischer Scientific), normalizing the infection rate of untreated virus controls as $100 \%$. 404 Half-maximal serum neutralizing titers (SNT ${ }_{50}$ ) were determined by curve fitting in 405 GraphPad Prism 8.

\section{Statistics}

407 Data were analyzed using GraphPad Prism 8. Results are represented as individual 408 values and median for summary statistics. Statistical significance was determined 409 using non-parametric Mann-Whitney U-test ( ${ }^{*} \mathrm{P} \leq 0.05 ;{ }^{*} \mathrm{P} \leq 0.01$; ns, not significant) 


\section{References}

413 1. Dallmeier, K., G. Meyfroidt, and J. Neyts, COVID-19 and the intensive care unit: vaccines to the rescue. Intensive Care Medicine, 2021: p. 1-4.

415

416

417

418

419

2. Heinz, F.X. and K. Stiasny, Distinguishing features of current COVID-19 vaccines: knowns and unknowns of antigen presentation and modes of action. npj Vaccines, 2021. 6(1): p. 1-13.

3. Verbeke, R., et al., The dawn of mRNA vaccines: The COVID-19 case. Journal of Controlled Release, 2021. 333: p. 511-520.

4. Neukirch, L., et al., The potential of adenoviral vaccine vectors with altered antigen presentation capabilities. Expert review of vaccines, 2020. 19(1): p. 2541.

5. Anderson, R.M., et al., Challenges in creating herd immunity to SARS-CoV-2 infection by mass vaccination. The Lancet, 2020. 396(10263): p. 1614-1616.

6. Garcia-Beltran, W.F., et al., Multiple SARS-CoV-2 variants escape neutralization by vaccine-induced humoral immunity. Cell, 2021. 184(9): p. 2372-2383. e9.

7. Cromer, D., et al., Neutralising antibody titres as predictors of protection against SARS-CoV-2 variants and the impact of boosting: a meta-analysis. The Lancet Microbe, 2021.

8. Cai, C., et al., The efficacy of COVID-19 vaccines against the B. 1.617.2 (delta) variant. Molecular Therapy, 2021. 29(10): p. 2890-2892.

9. Lai, C.-C., et al., COVID-19 vaccines: concerns beyond protective efficacy and safety. Expert Review of Vaccines, 2021. 20(8): p. 1013-1025.

10. Cai, C., et al., A comprehensive analysis of the efficacy and safety of COVID19 vaccines. Molecular Therapy, 2021. 29(9): p. 2794-2805.

11. Rosenblum, H.G., et al., Use of COVID-19 vaccines after reports of adverse events among adult recipients of Janssen (Johnson \& Johnson) and mRNA COVID-19 vaccines (Pfizer-BioNTech and Moderna): update from the Advisory Committee on Immunization Practices-United States, July 2021. Morbidity and Mortality Weekly Report, 2021. 70(32): p. 1094.

12. Lai, C.-C., et al., COVID-19 vaccines and thrombosis with thrombocytopenia syndrome. Expert Review of Vaccines, 2021. 20(8): p. 1027-1035.

13. Tobaiqy, M., et al., Thrombotic adverse events reported for Moderna, Pfizer and Oxford-AstraZeneca COVID-19 vaccines: comparison of occurrence and clinical outcomes in the EudraVigilance database. Vaccines, 2021. 9(11): p. 1326.

14. Schultz, N.H., et al., Thrombosis and thrombocytopenia after ChAdOx1 nCoV19 vaccination. New England journal of medicine, 2021. 384(22): p. 2124-2130.

15. Witberg, G., et al., Myocarditis after Covid-19 vaccination in a large health care organization. New England Journal of Medicine, 2021.

16. Bonaldo, M.C., P.C. Sequeira, and R. Galler, The yellow fever 17D virus as a platform for new live attenuated vaccines. Human vaccines \& immunotherapeutics, 2014. 10(5): p. 1256-1265.

17. Sanchez-Felipe, L., et al., A single-dose live-attenuated YF17D-vectored SARS-CoV-2 vaccine candidate. Nature, 2021. 590(7845): p. 320-325.

18. Hansen, C.A. and A.D. Barrett, The Present and Future of Yellow Fever Vaccines. Pharmaceuticals, 2021. 14(9): p. 891. 
19. Monath, T.P., et al., Live virus vaccines based on a yellow fever vaccine backbone: standardized template with key considerations for a risk/benefit assessment. Vaccine, 2015. 33(1): p. 62-72.

20. Barrett, A.D. and D.E. Teuwen, Yellow fever vaccine-how does it work and why do rare cases of serious adverse events take place? Current opinion in immunology, 2009. 21(3): p. 308-313.

21. de Lataillade, L.d.G., et al., Risk of yellow fever virus transmission in the AsiaPacific region. Nature communications, 2020. 11(1): p. 1-10.

22. McGee, C.E., et al., Substitution of Wild-Type Yellow Fever Asibi Sequences for 17D Vaccine Sequences in ChimeriVax-Dengue 4 Does Not Enhance Infection of Aedes aegypti Mosquitoes. The Journal of infectious diseases, 2008. 197(5): p. 686-692.

23. Danet, L., et al., Midgut barriers prevent the replication and dissemination of the yellow fever vaccine in Aedes aegypti. PLoS neglected tropical diseases, 2019. 13(8): p. e0007299.

24. Julander, J.G., Animal models of yellow fever and their application in clinical research. Current opinion in virology, 2016. 18: p. 64-69.

25. Boudewijns, R., et al., STAT2 signaling restricts viral dissemination but drives severe pneumonia in SARS-CoV-2 infected hamsters. Nature communications, 2020. 11(1): p. 1-10.

26. Siddharthan, V., et al., Zika virus infection of adult and fetal STAT2 knock-out hamsters. Virology, 2017. 507: p. 89-95.

27. Monath, T.P., et al., A live, attenuated recombinant West Nile virus vaccine. Proceedings of the National Academy of Sciences, 2006. 103(17): p. 6694-6699.

28. Quaresma, J.A., et al., Immunity and immune response, pathology and pathologic changes: progress and challenges in the immunopathology of yellow fever. Reviews in medical virology, 2013. 23(5): p. 305-318.

29. Domingo, C., et al., Detection of yellow fever 17D genome in urine. Journal of clinical microbiology, 2011. 49(2): p. 760-762.

30. Hernandez, N., et al., Inherited IFNAR1 deficiency in otherwise healthy patients with adverse reaction to measles and yellow fever live vaccines. The Journal of experimental medicine, 2019. 216(9): p. 2057-2070.

31. Franz, A.W., et al., Tissue barriers to arbovirus infection in mosquitoes. Viruses, 2015. 7(7): p. 3741-3767.

32. Kramer, L.D. and A.T. Ciota, Dissecting vectorial capacity for mosquito-borne viruses. Current opinion in virology, 2015. 15: p. 112-118.

33. Pulendran, B., Learning immunology from the yellow fever vaccine: innate immunity to systems vaccinology. Nature Reviews Immunology, 2009. 9(10): p. 741-747.

34. Appaiahgari, M.B. and S. Vrati, Clinical development of IMOJEV®-a recombinant Japanese encephalitis chimeric vaccine (JE-CV). Expert opinion on biological therapy, 2012. 12(9): p. 1251-1263.

35. Guy, B., et al., A recombinant live attenuated tetravalent vaccine for the prevention of dengue. Expert review of vaccines, 2017. 16(7): p. 671-684.

36. Biedenbender, R., et al., Phase II, randomized, double-blind, placebo-controlled, multicenter study to investigate the immunogenicity and safety of a West Nile virus vaccine in healthy adults. Journal of Infectious Diseases, 2011. 203(1): p. 75-84. 
37. Kum, D.B., et al., A chimeric yellow fever-Zika virus vaccine candidate fully protects against yellow fever virus infection in mice. Emerging microbes \& infections, 2020. 9(1): p. 520-533.

38. Bonaldo, M.C., et al., Recombinant yellow fever vaccine virus 17D expressing simian immunodeficiency virus SIVmac239 gag induces SIV-specific CD8+ Tcell responses in rhesus macaques. Journal of virology, 2010. 84(7): p. 36993706.

39. Bredenbeek, P.J., et al., A recombinant Yellow Fever 17D vaccine expressing Lassa virus glycoproteins. Virology, 2006. 345(2): p. 299-304.

40. Boudewijns, R., et al., A novel therapeutic hepatitis B vaccine candidate induces strong polyfunctional cytotoxic T cell responses in mice. JHEP Reports, 2021: p. 100295.

41. Monath, T.P., Review of the risks and benefits of yellow fever vaccination including some new analyses. Expert review of vaccines, 2012. 11(4): p. 427448.

42. Lindsey, N.P., et al., Adverse event reports following yellow fever vaccination, 2007-13. Journal of travel medicine, 2016. 23(5): p. taw045.

43. Bastard, P., et al., Auto-antibodies to type I IFNs can underlie adverse reactions to yellow fever live attenuated vaccine. Journal of Experimental Medicine, 2021. 218(4).

44. Schoggins, J.W., Interferon-stimulated genes: what do they all do? Annual review of virology, 2019. 6: p. 567-584.

45. Fan, Z., et al., Efficient gene targeting in golden Syrian hamsters by the CRISPR/Cas9 system. PloS one, 2014. 9(10): p. e109755.

46. Klitting, R., et al., Molecular determinants of yellow fever virus pathogenicity in Syrian golden hamsters: one mutation away from virulence. Emerging microbes \& infections, 2018. 7(1): p. 1-18.

47. Vazeille-Falcoz, M., et al., Variation in oral susceptibility to dengue type 2 virus of populations of Aedes aegypti from the islands of Tahiti and Moorea, French Polynesia. The American journal of tropical medicine and hygiene, 1999. 60(2): p. 292-299. 


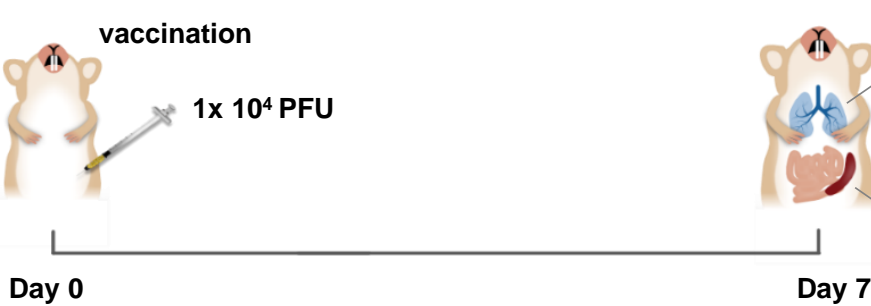

group 1: WT hamsters, sham (neg control) $(n=4)$

group 2: WT hamsters, YF17D ( $n=6)$

Day 7
Organ collected:

$\begin{array}{ll}\text { Spleen } & \text { Kidney } \\ \text { Liver } & \text { lleum } \\ \text { Brain } & \text { Parotid gland } \\ & \text { Lung }\end{array}$

group 3: WT hamsters, YF-S0 $(n=6)$

group 4: STAT2-/- hamsters, YF17D (pos control) $(\mathrm{n}=2)$

(B)

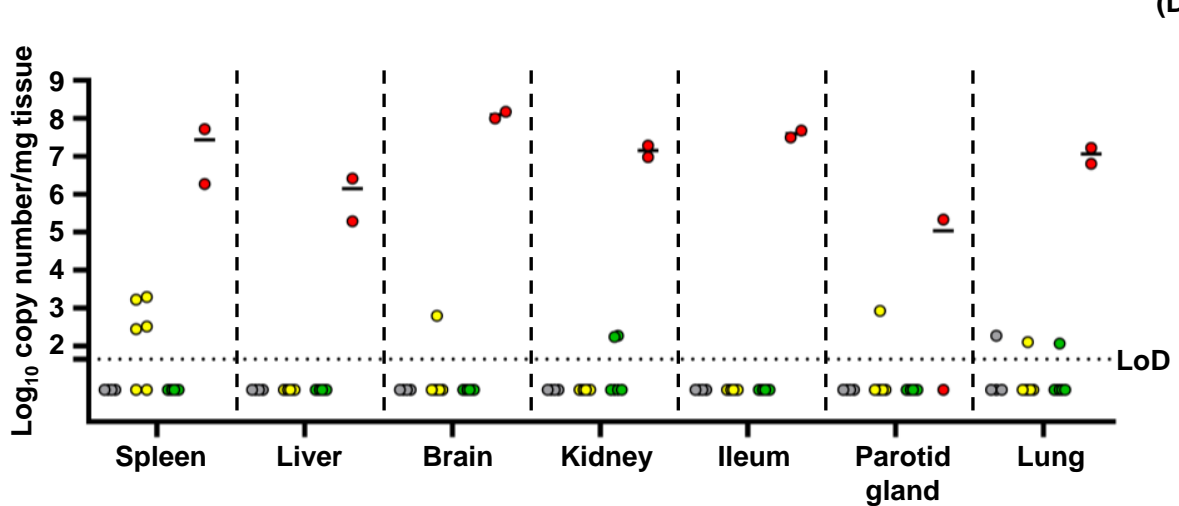

(D)

(\%)

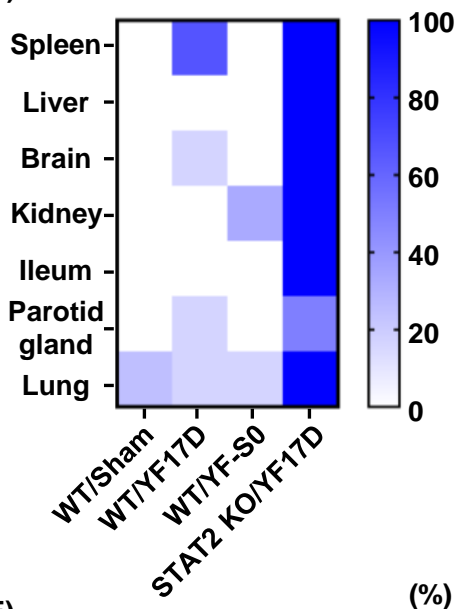

(C)

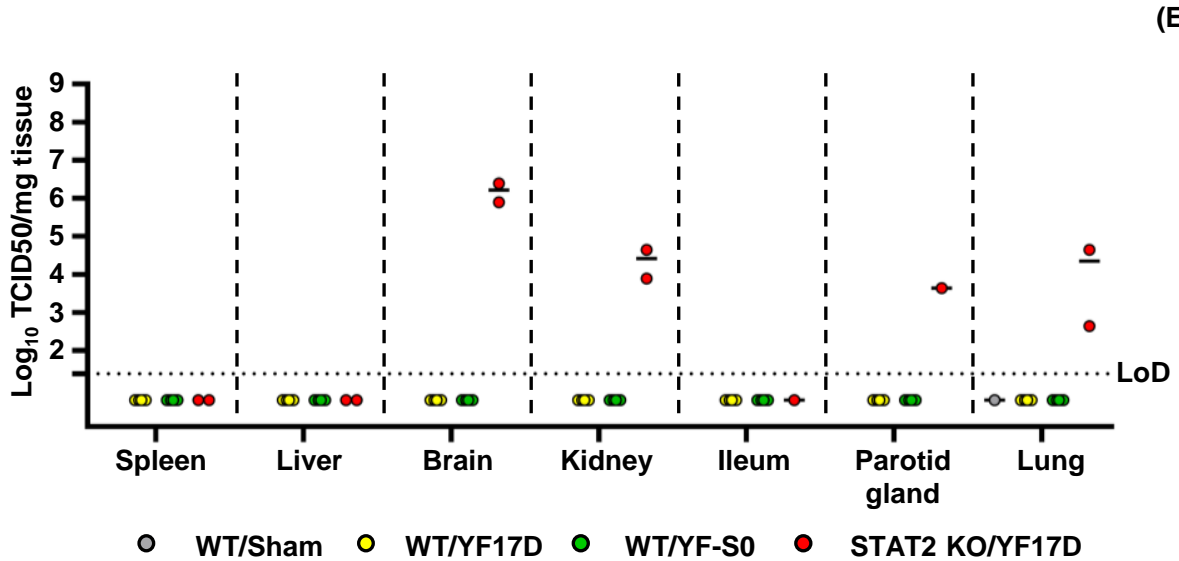

(E)

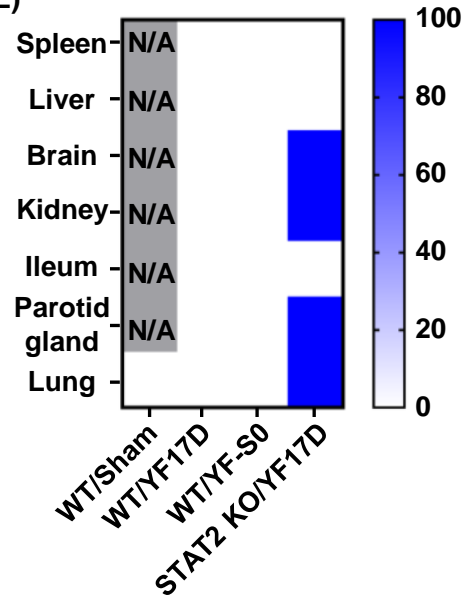

Fig. 1. Biodistribution of YF-S0 in hamsters. (A) Schematic of hamster vaccination and organ collection. Hamsters were inoculated i.p. with $10^{4} \mathrm{pfu} / \mathrm{ml}$ of either YF17D or YF-S0 and sacrificed 7 days later. Organs from 4 different experimental groups, including Sham vaccinated wild-type (WT) hamsters and YF17D vaccinated STAT2 ${ }^{-/}$knock out (KO) hamsters as respective negative and positive controls, were collected and divided for RNA extraction and virus isolation. (B) Viral RNA load by RT-qPCR. (C) Virus isolation by TCID $_{50}$ assay. For Sham and STAT2 KO, only PCR-positive samples were analyzed. (D,E) Heat map representing positivity rates by organ and experimental group based on the results of RT-qPCR (D) or TCID50 assay (E). Bars in (B) and (C) represent median values. $\mathrm{N} / \mathrm{A}$ : not applicable 
(A)

\section{$\begin{array}{lccccccc} & & \mid & \mid & & \mid & \mid \\ \text { D0 } & \text { D1 } & \text { D2 } & \text { D3 } & \text { D4 } & \text { D5 } & \text { D7 } & \text { D9 }\end{array}$ group 1: WT hamsters, sham (neg control) $(n=4)$ group 2: WT hamsters, YF17D $(n=6)$}

(B)

(C)

(D)

(E)

$\underline{\text { Urine }}$

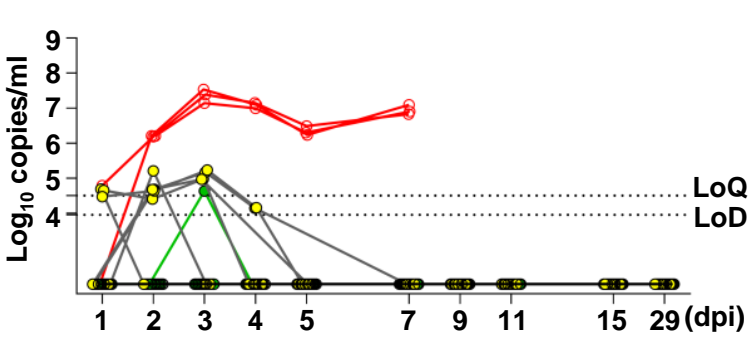

(F)

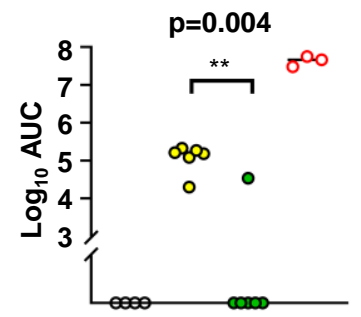

(G)
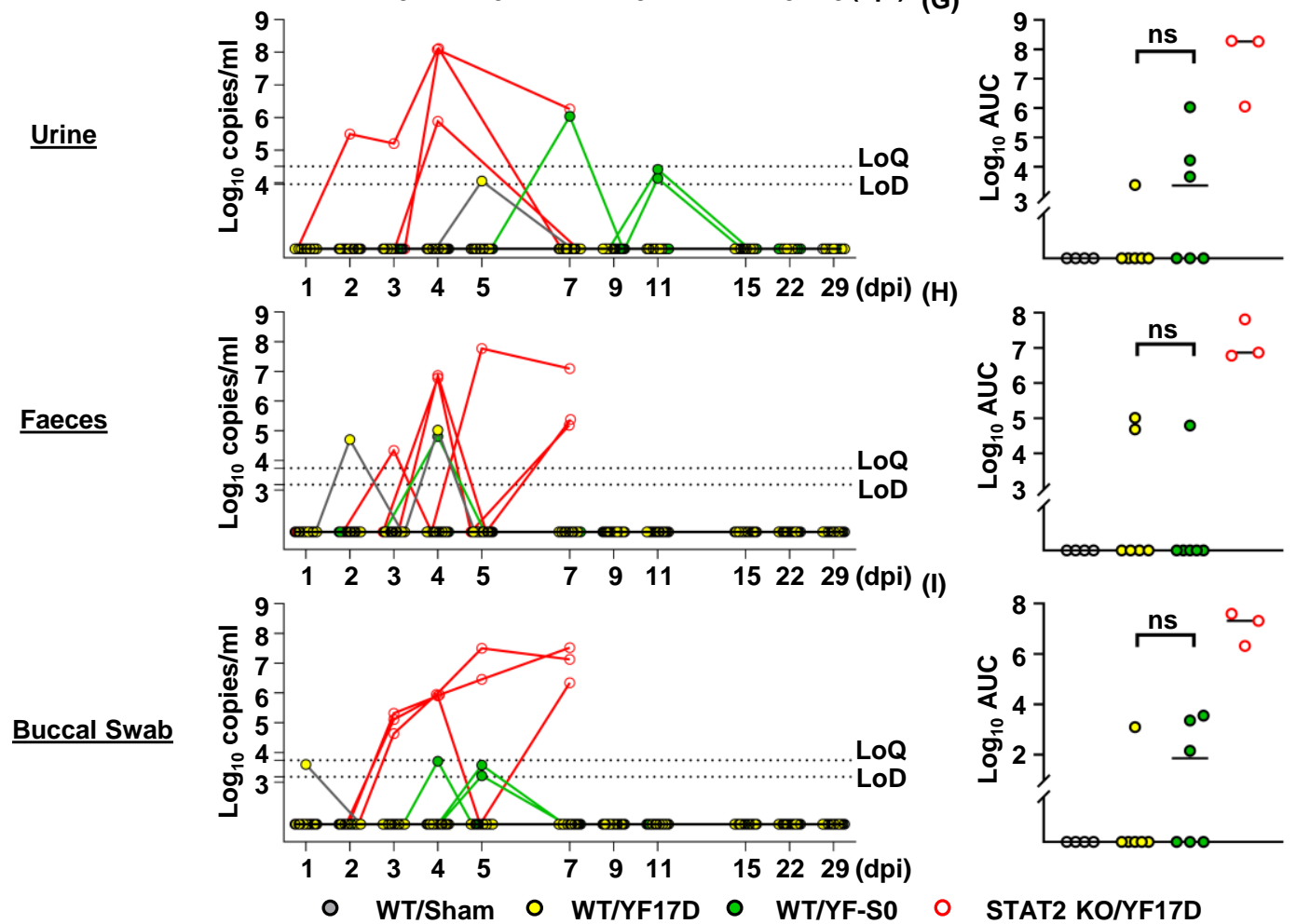

Fig. 2. Shedding of YF-SO by vaccinated hamsters. (A) Schematic of vaccination and specimens' collection. Hamsters were inoculated as in Fig. 1A, and serum, urine, faeces, and buccal swabs serially sampled at indicated timepoints. (B)-(E) Viral RNA load by RTqPCR. (F)-(I) Area under curve (AUC, copies*day) calculated by GraphPad Prism 8; Mann-Whitney test was used for the statistic analysis, with $p>0.05$ marked as nonsignificant (ns), and $p \leq 0.01$ as **. Serum RNA data for YF17D and YF-S0 vaccinated WT hamsters as previously published. LoQ: Limit of quantification; LoD: Limit of detection; dpi: days post inoculation. 
(A)

Day 0 :

Feeding

Mock $\quad(n=21) \quad 45$ mins

YF17D $\quad(n=20)$

YF-SO $\quad(n=25)$

YF-Asibi $(n=28)$
Day 0:

A: Ingestion Check (whole mosquito)

Mock $(n=5)$

YF17D $(n=5)$

YF-S0 $\quad(n=5)$

YF-Asibi $(n=5)$
Day 14:

B: Infection Check (dash area: main body)

Mock $\quad(n=16)$

YF17D $\quad(n=15)$

YF-S0 $\quad(n=20)$

YF-Asibi $(n=23)$

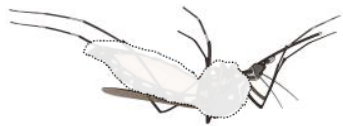

Day 14:

C: Dissemination Check (head, legs, and wings)

YF17D $\quad(n=6)$

YF-S0 $\quad(n=6)$

YF-Asibi $(n=6)$
(B)

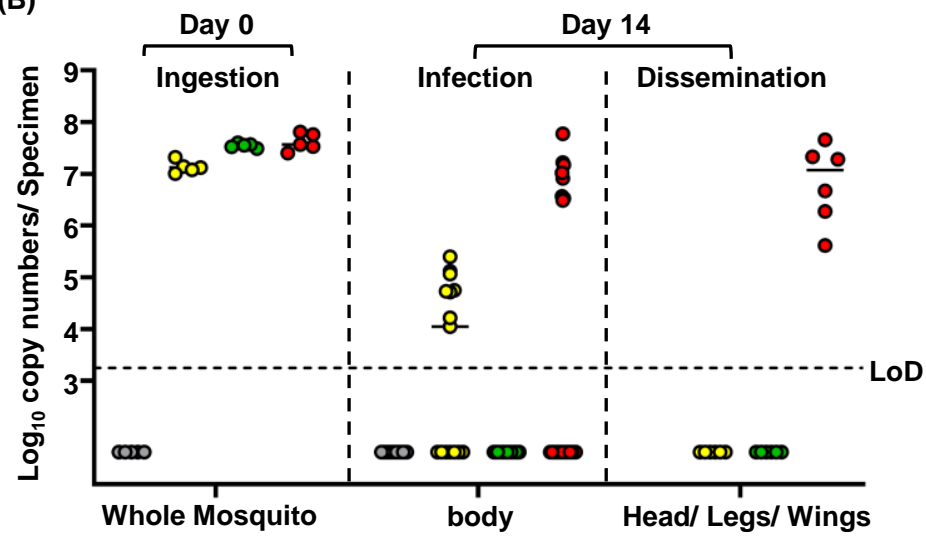

(C)

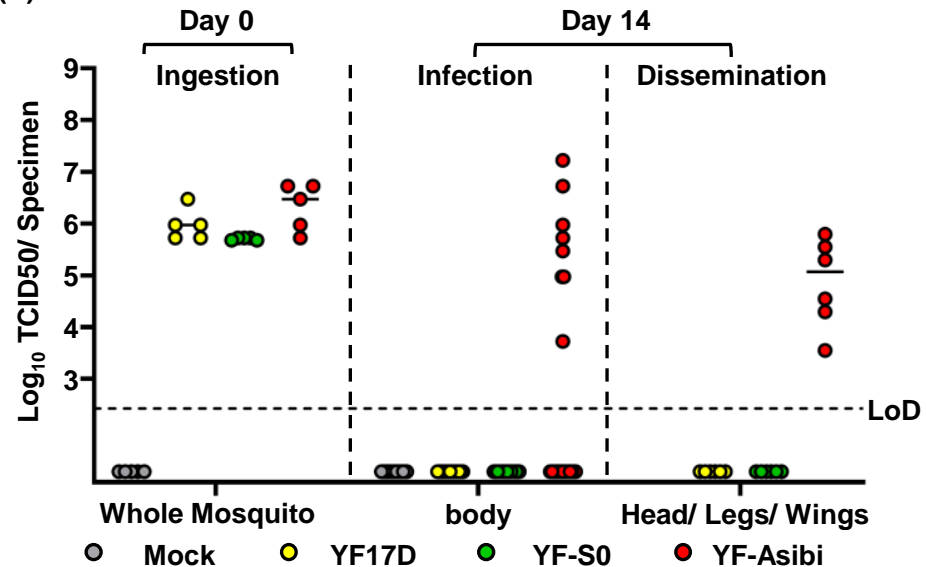

(D)

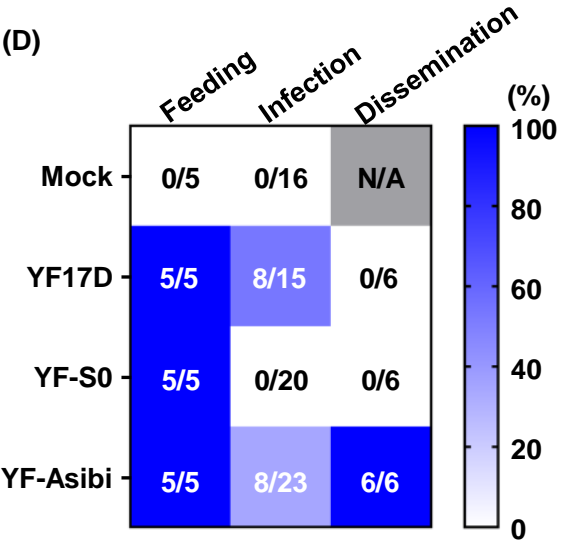

(E)

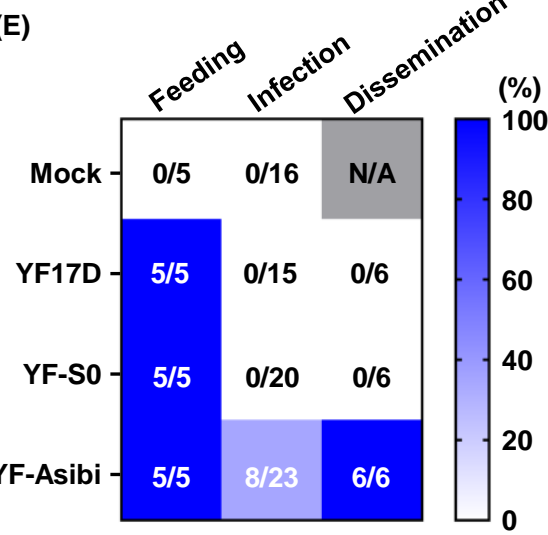

Fig. 3. Assessment of YF-SO transmission potential by Aedes mosquitoes. (A) Schematic of virus feeding of mosquitoes and specimens' collection. Mosquitoes were fed with infectious blood meal containing YF17D, YF-S0 or YF-Asibi, or Mock. 5 mosquitoes were collected each for ingestion assessment. At 14 days post feeding (dpf), remaining mosquitoes were dissected into two parts, midgut (infection assessment) and head, legs, and wings (dissemination assessment). (B) Viral RNA load by RT-qPCR. (C) Virus isolation by TCID50 assay. For assessment of ingestion and infection, RT-qPCR and TCID50 were performed on all samples. For assessment of dissemination, only a selection of PCR-positive specimens from the YF17D and YF-Asibi groups ( $n=6$ each) were further analyzed by TCID50 assay, plus 6 randomly chosen from the YF-S0 group. (D\&E) Heat map representing positivity rates per experiment group as scored by RT-qPCR (D) and TCID50 assay (E). Bars in (B) and (C) represent median values. N/A: not applicable. Mosquito icons were adapted from BioRender.com (2021). 

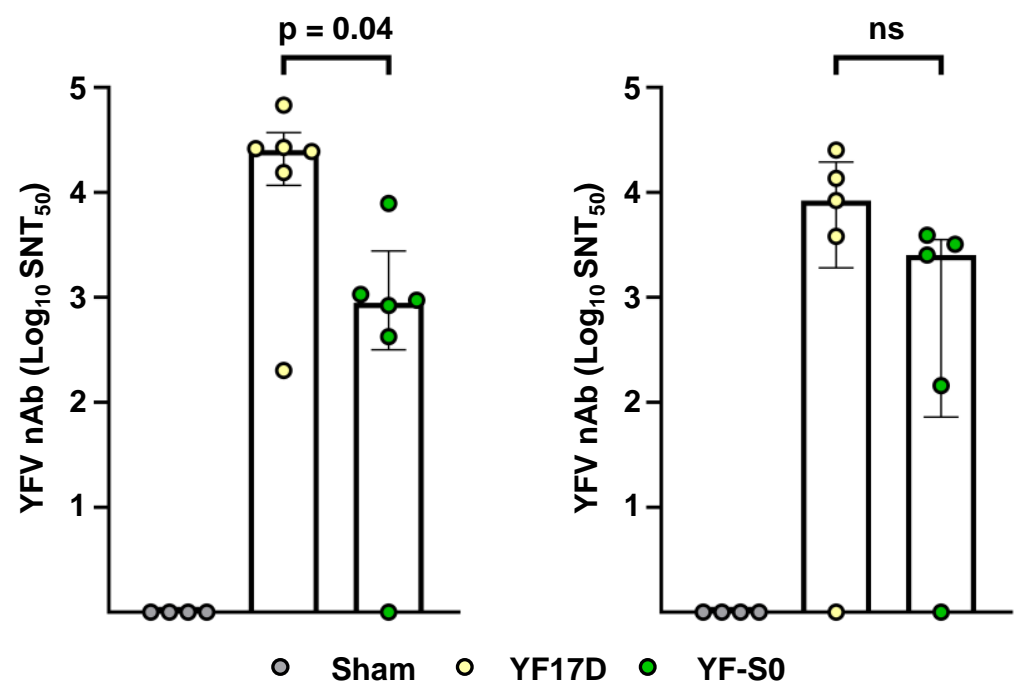

YF17D $\circ$ YF-S0

Fig. S1 (related to Fig. 1A and Fig. 2A). YFV-specific humoral immune responses in one-dose YF17D and YF-S0 vaccinated WT hamsters (primary pharmacodynamics). Serum samples for determination of YFV-specific neutralizing antibodies (nAb) collected at the respective endpoint of experiments assessing vaccine virus biodistribution (Fig.1A, 7 dpi) and shedding (Fig. 2A, 29 dpi). Sample number in biodistribution experiment: Sham $n=4$, YF17D $n=6$, and YF-S0 $n=6$, and in shedding experiment: Sham $n=4$, YF17D $n=5$, and YF-S0 $n=5$. 50\% serum neutralizing titers $\left(\mathrm{SNT}_{50}\right)$ were presented as median \pm IQR for each group at logarithmic scale. MannWhitney test was used for the statistical analysis, with $p>0.05$ marked as non-significant (ns). 


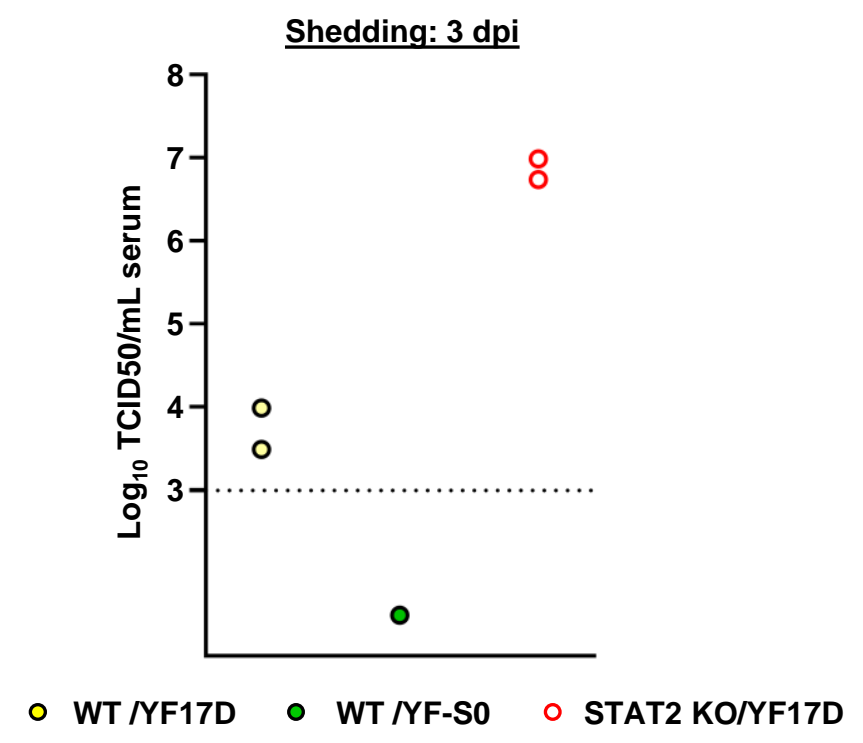

Fig. S2 (related to Fig. 2A). Infectious virus loads in serum (viremia) in selected YF17D or YF-S0 vaccinated hamsters. Serum samples collected at 3 days after vaccination. Sample number for YF17D vaccinated WT hamster, $n=2$; for YF-S0 vaccinated WT hamster, $n=1$; and (3) YF17D vaccinated STAT2 ${ }^{-/}$hamster, $n=2$. Selected samples included specimen with respectively highest viral RNA copies numbers detected (YF17D in WT and STAT2 ${ }^{--}$hamsters) or, in case of YF-S0, the only PCR positive specimen. 


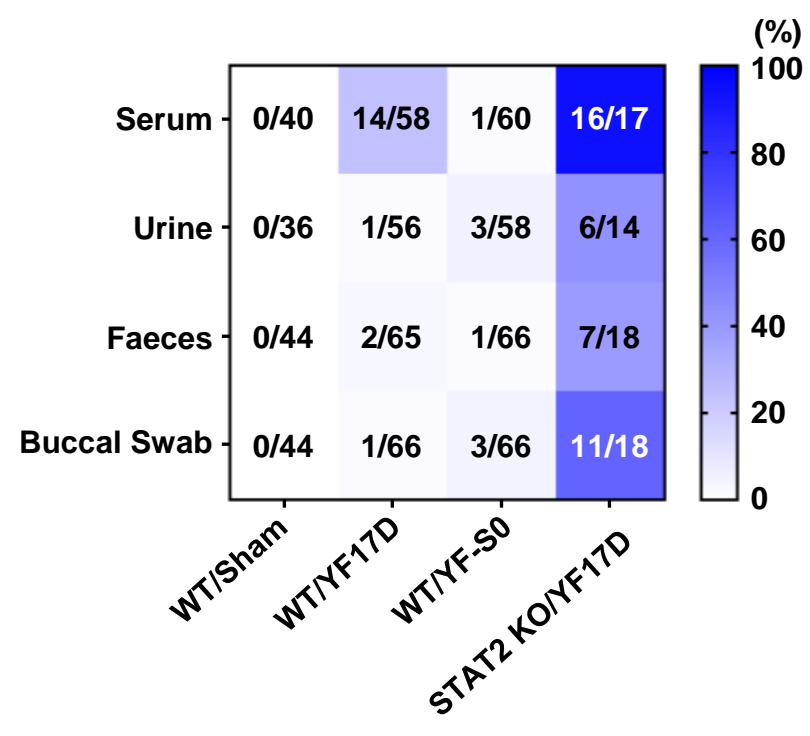

Fig. S3 (related to Fig. 2A). Cumulative detection rates of viral RNA by RT-qPCR in all specimens (serum, urine, faeces or buccal swab) collected from vaccinated WT or STAT2 ${ }^{-/}$hamster. Specimens were collected according to sampling scheme depicted in Fig. 2A. Heatmap showing ratios of the total number of all PCR-positive samples versus the total number of all samples tested per study group over the course of 29 days after vaccination. 